Check for updates

Cite this: RSC Adv., 2019, 9, 20281

Received 16th March 2019

Accepted 8th June 2019

DOI: 10.1039/c9ra02039j

rsc.li/rsc-advances

\section{Investigation of synergistic effects between silica nanoparticles, biosurfactant and salinity in simultaneous flooding for enhanced oil recovery}

\author{
Rasoul Khademolhosseini, (D) a Arezou Jafari, *a Seyyed Mohammad Mousavi*b \\ and Mehrdad Manteghian ${ }^{a}$
}

The purpose of this study was to investigate the effect of process parameters including silica nanoparticle (NP) concentration, biosurfactant (BS) concentration, and salinity as well as their synergistic effects on oil recovery in simultaneous flooding. Additionally, the effect of NP morphology (in the BS-NP solution) on oil recovery was investigated in this research. Micromodel flooding tests were designed with a central composite design (CCD) and carried out using BS and spherical silica NPs. The results showed that there is a positive synergistic effect between BS and silica NPs to shift the wettability to the water-wet condition and decrease interfacial tension (IFT), resulting in improved oil recovery. Indeed, the maximum oil recovery was obtained at an optimum salt concentration. Several micromodel tests were then carried out with BS and different-shaped NPs at the optimum point predicted by a mathematical model to study the effect of NP morphology on oil recovery. The results showed that minimum IFT of $1.85 \mathrm{mN} \mathrm{m}^{-1}$ and the most reduction in the glass contact angle of $92.8 \%$ could be achieved by the BS-spherical NP solution as compared to those of the BS-non spherical NP solutions, which led to the highest oil recovery of $53.4 \%$. The better performance of spherical NPs was attributed to the higher uniformity, which resulted in better distribution and more effective interactions with crude oil components. The results of core flooding tests showed that the BS-spherical NP solution yielded $26.1 \%$ final oil recovery after brine flooding. In addition, the BS-NP solution was more effective in wettability alteration of an oilwet carbonate rock compared with the BS solution. It was deduced that the main mechanisms involved in oil recovery improvement were wettability alteration to the water-wet state, IFT reduction, and mobility ratio improvement.

\section{Introduction}

The traditional methods of oil recovery from oil reservoirs have been categorized as primary and secondary methods. Primary oil recovery is the first stage of oil production, in which the reservoir pressure is the driving force of the natural rise of crude oil. Water flooding is generally applied in the secondary recovery stage to displace the trapped oil in the rock towards production. However, these stages are not efficient enough to produce a considerable amount of oil due to high capillary forces, which result in low oil recovery. ${ }^{1-3}$ The methods to further increase the sweep efficiency and enhance oil recovery comprise tertiary recovery or enhanced oil recovery (EOR). Nowadays, applying new methods such as biotechnology and nanotechnology in EOR has aroused much attention.

${ }^{a}$ Faculty of Chemical Engineering, Tarbiat Modares University, Tehran, Iran. E-mail: ajafari@modares.ac.ir; Fax: +98-21-82884931; Tel: +98-21-82884982

${ }^{b}$ Biotechnology Group, Chemical Engineering Department, Tarbiat Modares University, Tehran, Iran. E-mail: mousavi_m@modares.ac.ir; Fax: +98-21-82884931; Tel: +98-2182884917
Numerous studies have been dedicated to the application of biosurfactants in EOR and the investigation of their ability in IFT reduction and wettability alteration, which are favorable in EOR. $^{4-7}$ Biosurfactant (BS) molecules diffuse from the bulk aqueous phase to the interface of the crude oil-aqueous phase due to the concentration gradient and then adsorb at the surface. Generally, diffusion is much slower than adsorption, thus controlling the whole process. ${ }^{8}$ The hydrophilic head and hydrophobic tail of biosurfactants interact with water molecules and crude oil, respectively. This forms a bridge between two phases and lowers IFT. IFT reduction decreases capillary forces in the pores and throats of porous media, which are the main reason for oil trapping, and improving oil recovery.,10 Khademolhosseini et al. ${ }^{11}$ synthesized a rhamnolipid BS from newly found Pseudomonas aeruginosa HAK01 that showed excellent ability in IFT reduction and wettability alteration of the glass surface from the oil-wet state to the water-wet state. It also showed high stability under severe conditions of temperature, $\mathrm{pH}$, and salinity, which is necessary to achieve good effectiveness in microbial enhanced oil recovery (MEOR). Indeed, the results obtained from micromodel tests confirmed that the 
produced BS is a good candidate in this field. Saxena et al. ${ }^{12}$ produced anionic BS from palm oil and studied its ability to alter the wettability of quartz and carbonate surfaces. The produced BS changed the wettability of both surfaces from the oil-wet state to the water-wet state.

Furthermore, surfactants and biosurfactants have other applications in other industrial fields due to their high surface activity. For example, they have been introduced in biofiltration systems to lower the surface tension; thus, the solubility of volatile organic compounds (VOCs) increases and consequently, higher removal efficiency of these compounds is achieved. ${ }^{\mathbf{9}, 13}$ There are studies on the application of biosurfactants (such as saponins and rhamnolipids) and nonionic chemical surfactants (such as Tween 20 and Triton X-100) for the removal of styrene, ethylbenzene, and $n$-hexane from the waste gas in biofilters. $^{9,13-15}$

Nowadays, nanotechnology has attracted much attention for applications in EOR to produce more oil from reservoirs. Numerous studies confirm that NPs can alter the wettability characteristics of rocks to more water-wet conditions, ${ }^{\mathbf{1 6}-18}$ reduce IFT, ${ }^{19,20}$ and function effectively in oil recovery. ${ }^{21,22}$ According to Ragab and Hannora, ${ }^{23}$ silica NPs improve oil recovery due to reduction in IFT and increase in the viscosity of the injected solution.

Different studies show that NPs along with surfactants can improve oil recovery by synergistic effects compared with pure surfactant flooding. ${ }^{24-27}$ Zargartalebi et $a .^{28}$ showed that the application of hydrophilic silica NPs along with sodium dodecyl sulfate (SDS) reduced IFT and enhanced oil recovery from sandstone rocks. The results achieved were consistent with those reported by Ahmadi and Shadizadeh. ${ }^{29}$ Nwidee et al. ${ }^{30}$ showed that the mixture of NPs and surfactants altered the wettability of the system to the water-wet state. Mingwei et al. ${ }^{31}$ used silica NPs and TX-100 (a non-ionic surfactant) to evaluate their synergistic effects on oil recovery. The results showed that silica NPs mixed with this surfactant were more capable of wettability alteration compared to a pure surfactant solution.

Despite considerable efforts in the application of surfactants, biosurfactants, NPs, and surfactants along with NPs in oil recovery, no attention has been paid to the simultaneous flooding of biosurfactants and NPs in this field. To fill this gap, simultaneous flooding of NPs and anionic BS was performed in this research and the effects of process variables (NP concentration, BS concentration, and salinity) on oil recovery and their interactions, which have not been evaluated yet, were thoroughly investigated. Flooding tests were designed with a central composite design (CCD) and were conducted in a micromodel. A rhamnolipid-type BS and spherical silica NPs were applied in the designed tests. The process was then optimized to achieve maximum oil recovery and the confirmation test was conducted. Then, several micromodel tests and contact angle measurements were carried out at optimum point to evaluate the effect of NP morphology (in BS-NP solution) on oil recovery for the first time. Subsequently, core flooding tests were conducted using two BS-NP solutions, which led to the highest oil recovery in the micromodel tests at the optimum point. Indeed, the viscosity values of the injected fluids and IFT between crude oil and the injected solutions were measured for the mechanistic investigation of oil recovery. Furthermore, the wettability state of the glass surface and carbonate rock was investigated for this reason.

\section{Materials and methods}

\subsection{Materials}

Anionic rhamnolipid BS (CMC of $120 \mathrm{ppm}$ ), produced from Pseudomonas aeruginosa HAK01, ${ }^{11}$ and hydrophilic silica NPs were applied in this research (Fig. 1). NPs were synthesized by neutralizing potassium silicate with hydrochloric acid in the presence of polyethylene glycol (PEG 4000). The SEM images of the produced NPs are given in Fig. 1, and the FTIR spectrum of spherical NPs (Fig. 1A) is given in Fig. 2. Sodium chloride (99.5\% purity, Tetra-Chem) was used to obtain the required salinity in solutions. The crude oil sample used in this investigation was obtained from the North Azadegan oil field in Iran (specific gravity of 0.937 , TAN of $0.46 \mathrm{mg} \mathrm{KOH} / \mathrm{mg}$ oil, asphaltene content of $10.12 \mathrm{wt} \%$ and viscosity of $242 \mathrm{cP}$ at $25{ }^{\circ} \mathrm{C}$ ). Methanol (99.8\%), sodium hydroxide (99\%), trichloromethyl silane (99\%), and toluene (99.9\%), supplied by Merck Chemical Co., were used to make the micromodel oil-wet. Distilled water, methanol, and toluene were applied for cleaning the micromodel and core sample.

\subsection{Solution preparation}

BS and sodium chloride were added to distilled water and stirred using a magnetic stirrer until they totally dissolved. Silica NPs were then added to the solution and stirred for $30 \mathrm{~min}$ at the rate of $400 \mathrm{rpm}$ for initial dispersion. Finally, the solution was sonicated for at least $1 \mathrm{~h}$ in an ultrasonic bath (400 watt, $20 \mathrm{kHz}$ ).

\subsection{EOR application}

2.3.1 Flooding tests. In order to investigate the performance of BS-NP solutions in enhanced oil recovery, several solutions of BS and NPs were prepared and injected into a five spot micromodel. Before performing flooding tests, the micromodel was made strongly oil-wet by the following procedure: it was totally saturated with $\mathrm{NaOH}$ solution for $1 \mathrm{~h}$ and then rinsed thoroughly with distilled water. Afterwards, it was dried in an oven at $200{ }^{\circ} \mathrm{C}$ for at least $15 \mathrm{~min}$. The model was then saturated with a solution of $98 \%$ toluene and $2 \%$ trichloromethylsilane for at least 5-10 min. The porous medium was then washed with methanol and dried in an oven at $100{ }^{\circ} \mathrm{C}$ for at least 1 h. ${ }^{27}$ Fig. 3a demonstrates a schematic representation of the experimental setup employed for the micromodel test. To carry out a flooding test, the oil-wet glass micromodel was saturated with oil, followed by the injection of the prepared BS-NP solution $\left(0.05 \mathrm{ml} \mathrm{h}^{-1}\right)$. The micromodel was placed horizontally in all the tests to avoid the gravity effect. All the tests were carried out at ambient conditions. During the tests, high-resolution pictures were taken (Canon EOS 7D) at various time intervals to investigate the fluid distribution in the 

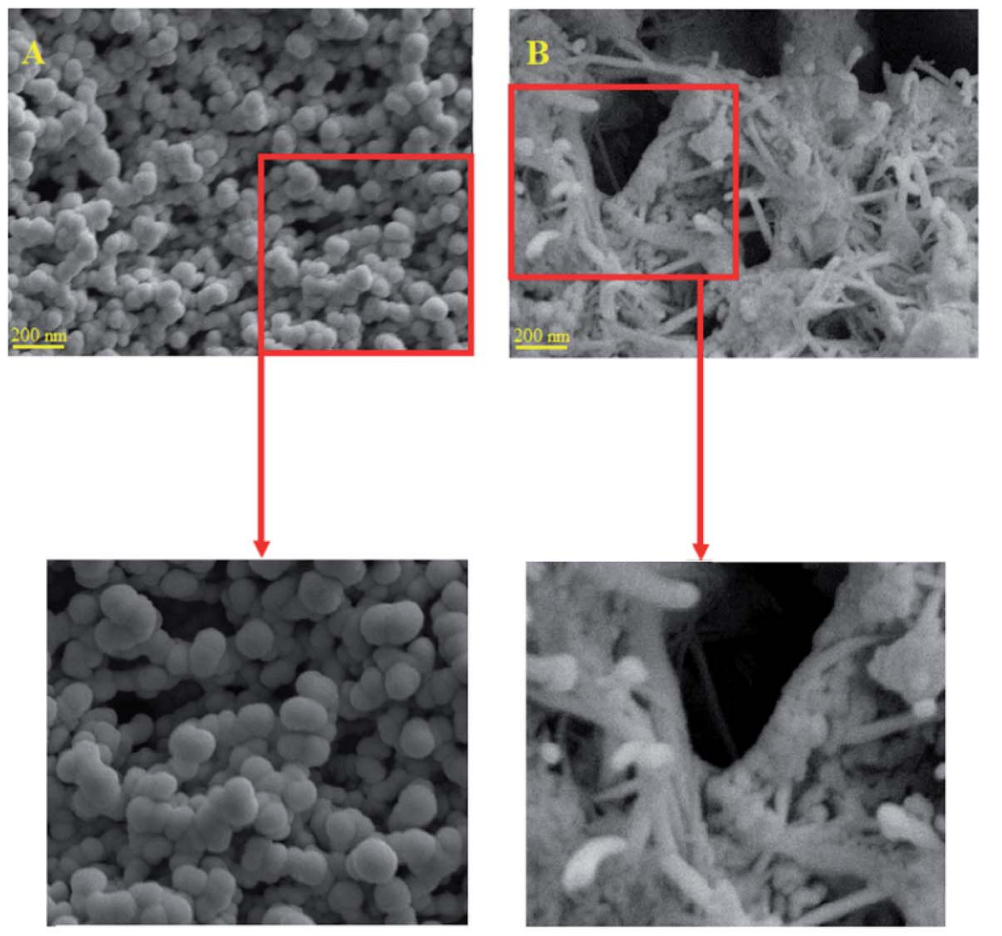
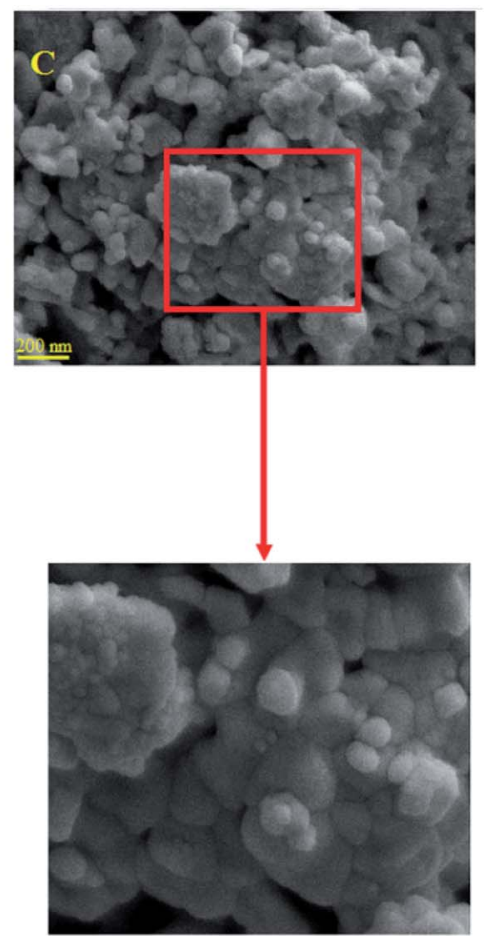
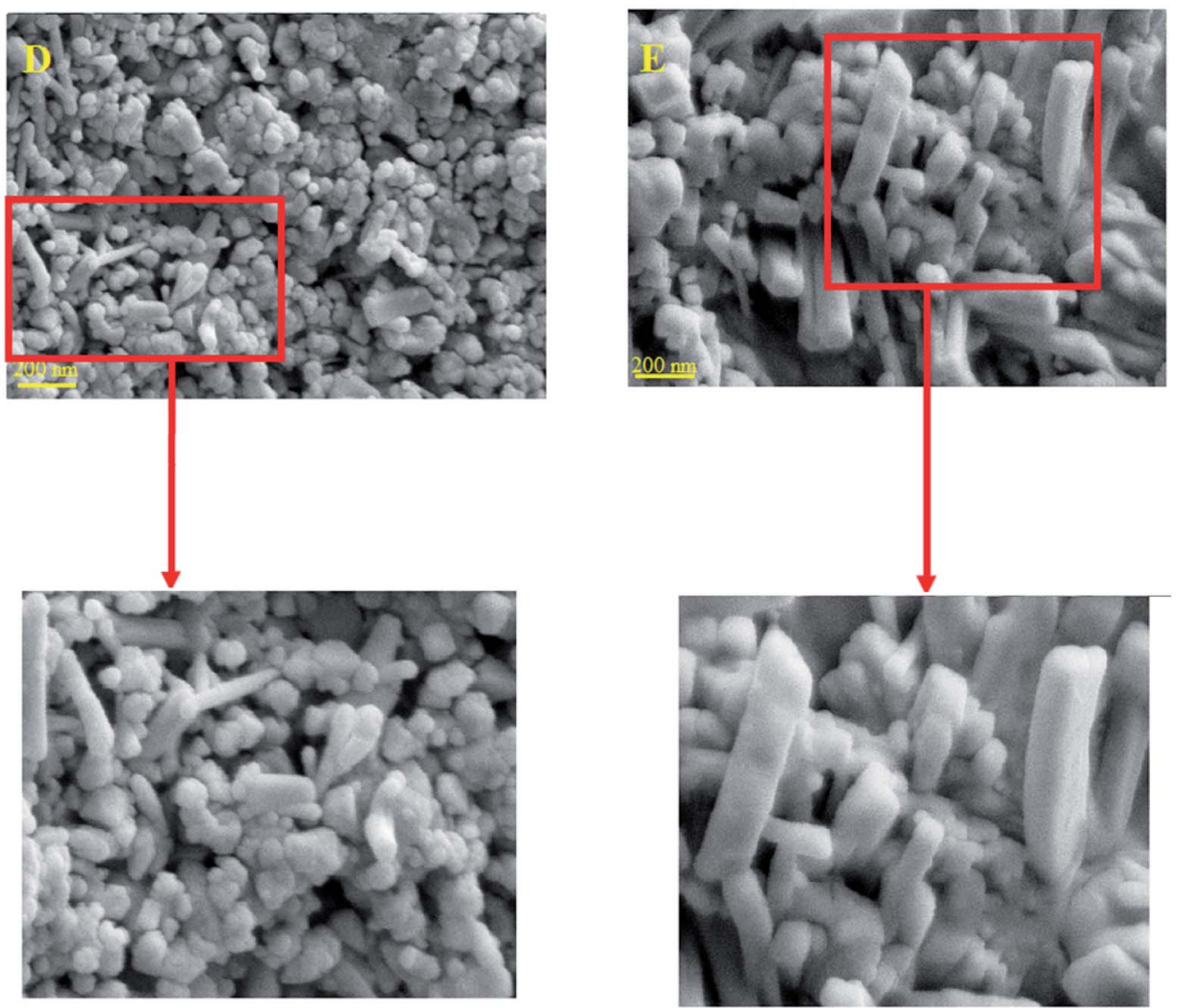

Fig. 1 SEM images of the synthesized NPs: (A) spherical $(78 \mathrm{~nm})$, (B) mixture of one-dimensional nanowires and irregular shapes (58 $\mathrm{nm})$, (C) sponge-like particles $(73 \mathrm{~nm})$, (D) mixture of spherical, rod-like and irregular particles $(44 \mathrm{~nm})$, and (E) rod-like particles with a tetragonal crosssection (92 $\mathrm{nm}$ ). 


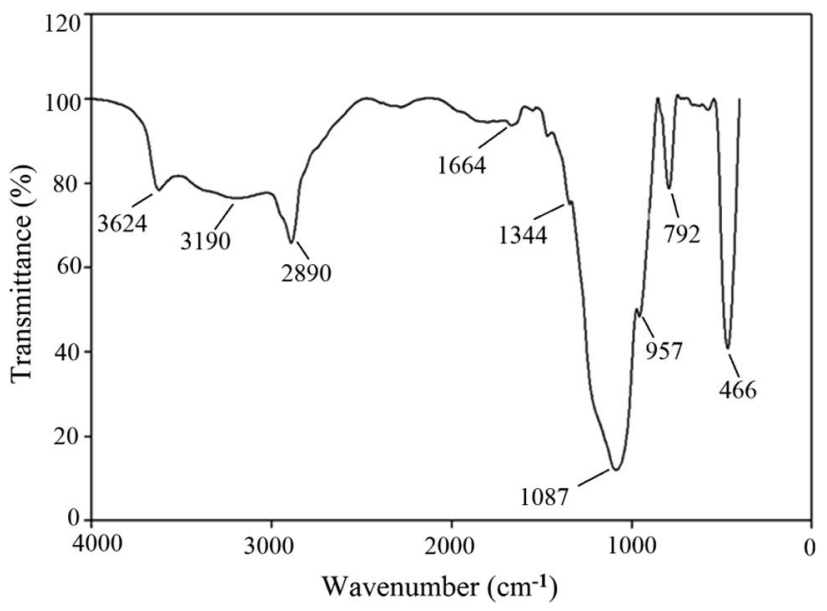

Fig. 2 The FTIR spectrum of spherical NPs.

micromodel. Final oil recovery was calculated using image analysis with Photoshop software (Adobe Photoshop CS6). ${ }^{11}$

Two core flooding tests were carried out to evaluate the effect of BS-NP solutions on oil recovery from a carbonate core sample. For this purpose, a carbonate core sample $3.74 \mathrm{~cm}$ in diameter and $6.2 \mathrm{~cm}$ in length was used in this research. The permeability and porosity of the used core sample were $1.45 \mathrm{mD}$ and 14.1, respectively. Initially, the core sample was washed with toluene and methanol, oven-dried, placed in a core flood, and subjected to reduced pressure for six hours using a vacuum pump. Subsequently, brine ( $3 \mathrm{wt} \% \mathrm{NaCl}$ ) was injected to establish initial water saturation. The crude oil was then injected until reaching irreducible water saturation $\left(S_{\mathrm{wi}}\right)$. Then, it was immersed in the crude oil and placed in the oven at $80^{\circ} \mathrm{C}$ for 15 days to change the wettability to the oil-wet state.

After aging, the brine solution ( $3 \mathrm{wt} \%$ sodium chloride) was injected, followed by the injection of BS-NP solution to study oil recovery from the carbonate rock. The values of oil production were recorded $v s$. time and finally, oil recovery was plotted $v s$. pore volumes of the injected solution. The setup used for the core flooding test is illustrated in Fig. $3 \mathrm{~b}$.

2.3.2 Wettability tests. Contact angle measurement is one of the conventional methods to investigate the wettability state

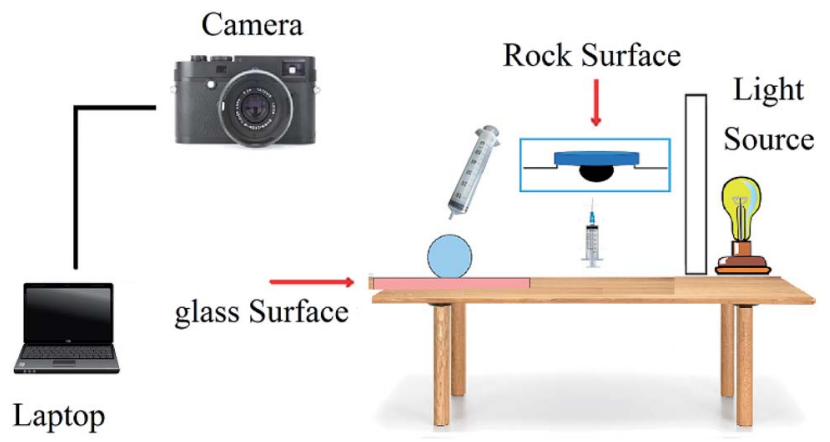

Fig. 4 Applied setup for contact angle measurement on glass surface and carbonate rock sample.

(hydrophilicity or hydrophobicity) of a solid surface. ${ }^{32-34}$ In this work, the static sessile-drop method was used to evaluate the effect of the solutions prepared on the wetting characteristics of the glass surface and a carbonate rock. ${ }^{32-34}$ For this purpose, small pieces of glass plates $(6 \mathrm{~cm} \times 4 \mathrm{~cm} \times 5 \mathrm{~mm})$ were used and made oil-wet based on the procedure described in 2.3.1 for the micromodel. Next, a droplet of the solution was placed on the oil-wet glass surface and then, droplet images were captured every two minutes. The variations of contact angle with time were plotted for the evaluation of wettability alteration. The smaller contact angle values indicate more hydrophilicity of the glass surface. ${ }^{7,32-34}$

The sessile drop method is the most common method to study the wettability state of a carbonate rock sample, ${ }^{27}$ and it was also applied in this research. For this purpose, pellets were taken apart from rock, washed with methanol, and aged with crude oil in an oven $\left(80^{\circ} \mathrm{C}\right)$ for two weeks in order to alter the wettability to oil-wet state. Afterwards, the oil-wet pellets were placed in solutions for a week to change the wettability to more water-wet state. Then, the flat surface of the pellet was placed horizontally in the solution, and a drop of oil was placed onto the pellet bottom. The contact angles were measured by imaging the droplets attached to the pellet (Fig. 4). Measurements were recorded for three different points on the pellet to obtain more accurate results and the average values were reported. Three solutions were considered for this test. Two of

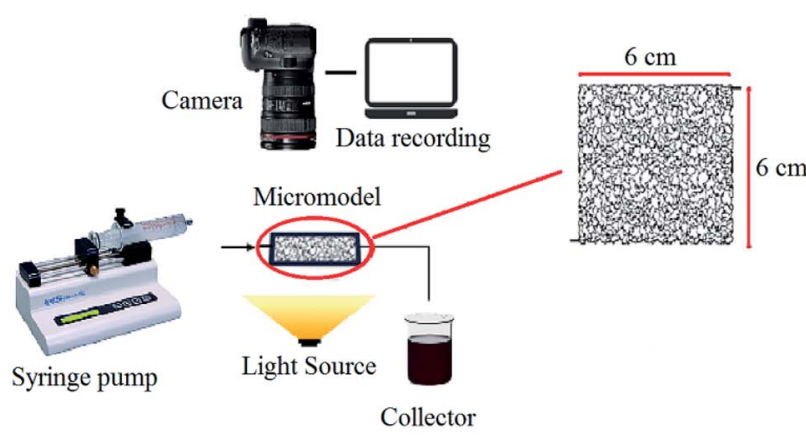

a

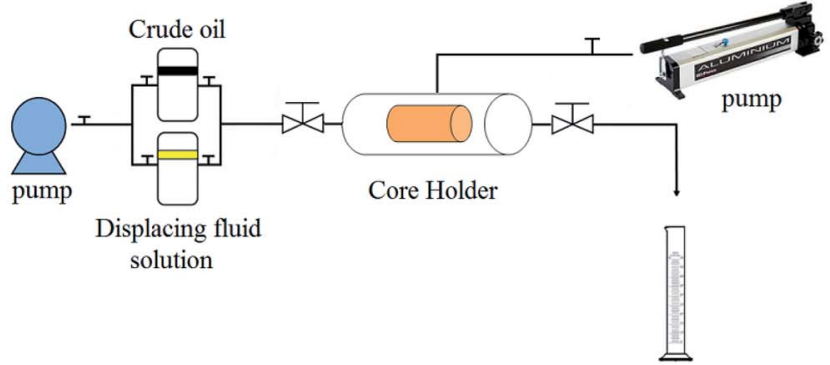

b

Effluent collector 
these solutions were in the optimum point condition of the micromodel tests but with NPs having different morphologies, which were applied in core tests. The other solution was in the optimum point condition too but without NPs to investigate the role of a pure BS solution in wettability alteration.

\subsection{Tests}

The viscosity of the injected solution affects the mobility ratio and therefore has an important role in the oil recovery process. In this work, a Brookfield viscometer (NDJ-4; China) was used to measure the viscosities of solutions at ambient temperature. The IFT values between crude oil and aqueous solutions were measured with a Pendant drop method using a VIT-ES30 apparatus (Fars EOR Technology Company). The zeta potential measurement of the solutions was performed using ZETAcheck, Particle Metrix (Germany) to evaluate the surface charge of BS and NPs. To have more reliable results, each experiment was carried out in triplicate, and the average values were reported.

\subsection{Designed experiments}

The central composite design method (CCD) is widely used as a statistical approach to analyze the effect of process variables on the response of the system including oil recovery efficiency in flooding experiments. ${ }^{11,35,36} \mathrm{In}$ this research, the micromodel flooding tests were designed to evaluate the interactions of operating variables and their effects on the final oil recovery. To do this, the CCD method was applied using the Design Expert software version 7.0. Three factors (NP concentration, BS concentration, and salinity) were considered as the main variables (Table 1). In order to maximize the oil recovery, process variables were optimized using mathematical and statistical tools and then, the confirmation test was performed.

\section{Results and discussion}

\subsection{Characterization of NPs}

Fig. 1 demonstrates the SEM images of NPs with different morphologies. Fig. 2 shows the FTIR spectrum of the produced spherical NPs. The peak at $466 \mathrm{~cm}^{-1}$ corresponds to the bending vibration of siloxane ( $\mathrm{Si}-\mathrm{O}-\mathrm{Si}$ ). The absorption peaks at 792 and $1087 \mathrm{~cm}^{-1}$ indicate the Si-O-Si symmetric and asymmetric stretching vibrations, respectively. The absorption peak at around $957 \mathrm{~cm}^{-1}$ is related to $\mathrm{Si}-\mathrm{OH}$ asymmetric stretching. The band at $1664 \mathrm{~cm}^{-1}$ is due to the bending vibration of the water molecules adsorbed on the surface of silica NPs. The

Table 1 Experimental parameters at different levels

\begin{tabular}{llccccccc}
\hline & & \multicolumn{5}{c}{ Level } \\
\cline { 5 - 8 } Factor & & Code & Unit & $-\alpha=-2$ & -1 & 0 & 1 & $+\alpha=2$ \\
\hline NP concentration & $\mathrm{A}$ & $(\mathrm{ppm})$ & 0 & 25 & 50 & 75 & 100 \\
BS concentration & $\mathrm{B}$ & $(\mathrm{ppm})$ & 0 & 40 & 80 & 120 & 160 \\
Salinity & $\mathrm{C}$ & $(\mathrm{wt} \%)$ & 0 & 0.5 & 1 & 1.5 & 2
\end{tabular}

broad peak observed at $3624 \mathrm{~cm}^{-1}$ is attributed to the $\mathrm{Si}-\mathrm{OH}$ stretching vibration and the $\mathrm{O}-\mathrm{H}$ stretching vibration of the water molecules adsorbed on the surface of silica by hydrogen bonding. These results indicate the presence of a silica network in the synthesized NPs.

The absorption peaks at $2890 \mathrm{~cm}^{-1}$ and $3190 \mathrm{~cm}^{-1}$ are associated with the symmetric and asymmetric stretching vibrations of alkyl groups, respectively. The absorption peak at $1344 \mathrm{~cm}^{-1}$ is due to the deforming vibration of alkyl groups. These three peaks confirm that PEG has been grafted onto the surface of silica NPs. ${ }^{37,38}$

\subsection{Designed micromodel flooding tests}

Table 2 shows the tests conducted under different conditions. Final oil recovery, breakthrough time (BT), and the viscosity values of injected fluids are also presented in this table.

3.2.1 Flooding tests at $0.5 \mathrm{wt} \%$ salinity. Fig. 5 shows the final oil recovery of tests $2,6,14$, and 18 versus NP/BS concentrations. All the mentioned solutions were flooded at $0.5 \mathrm{wt} \%$ salinity. The BS concentrations of the solutions of tests 2 and 6 were equal, $40 \mathrm{ppm}$, but the NP concentration in the solution of test $2(75 \mathrm{ppm})$ was thrice as compared to that of the solution of test $6(25 \mathrm{ppm})$. This resulted in oil recovery enhancement from $26.5 \%$ (test 6 ) to $40 \%$ (test 2 ); in simpler words, $13.5 \%$ more oil was recovered during test 2 .

The solutions of tests 14 and 18 exhibited equal BS concentration of $120 \mathrm{ppm}$. The concentration of NPs in the solution of test $14(75 \mathrm{ppm})$ was three times greater than that of the solution of test $18(25 \mathrm{ppm})$, leading to $7.5 \%$ more oil recovery: oil recovery increased from $42 \%$ (test 18 ) to $49.5 \%$ (test 14). The obtained results show that NPs have a positive effect on oil recovery, especially at lower BS concentrations.

Similarly, the BS concentration in solutions 18 and $14(120$ ppm) was three times greater than that of solutions 6 and 2 (40 $\mathrm{ppm}$ ), yielding $9.5 \%$ and $15.5 \%$ more recoveries, respectively: the oil recovery was enhanced from $40 \%$ (test 2) to $49.5 \%$ (test 14 ) and improved from $26.5 \%$ (test 6 ) to $42 \%$ (test 18 ).

The comparison of pair tests $(6,18)$ and $(2,14)$ shows that increasing the BS concentration strongly affects oil recovery, which is more effective at lower NP concentrations.

At lower NP concentrations, the interface was not saturated with NPs and therefore, BS molecules could easily diffuse into the interface and decrease the IFT value between the crude oil and aqueous phase. When the NP concentration increased, NPs accumulated at the interface more; as a result, it was more difficult for BS molecules to diffuse into the interface. Therefore, fewer BS molecules were adsorbed at the interface and they were less effective at higher NP concentrations. A closer look at the final oil recovery differences $(13.5 \%, 7.5 \%, 15.5 \%$, and $9.5 \%$ ) indicated that the increase in BS concentration was more effective than that in NP concentration.

3.2.2 Flooding tests at $\mathbf{1} \mathbf{w t} \%$ salinity. According to Table 2, the fluids injected in tests 10,11,16, and 17 (central points) as well as in 4 and 9 have equal salinity and BS concentration (1wt\% and $80 \mathrm{ppm}$, respectively); however, they differ in NP concentrations, which are 0,50 , and $100 \mathrm{ppm}$ for test 9 , central 
Table 2 Experimental design factors, final oil recovery, BT, and viscosities of the injected fluids

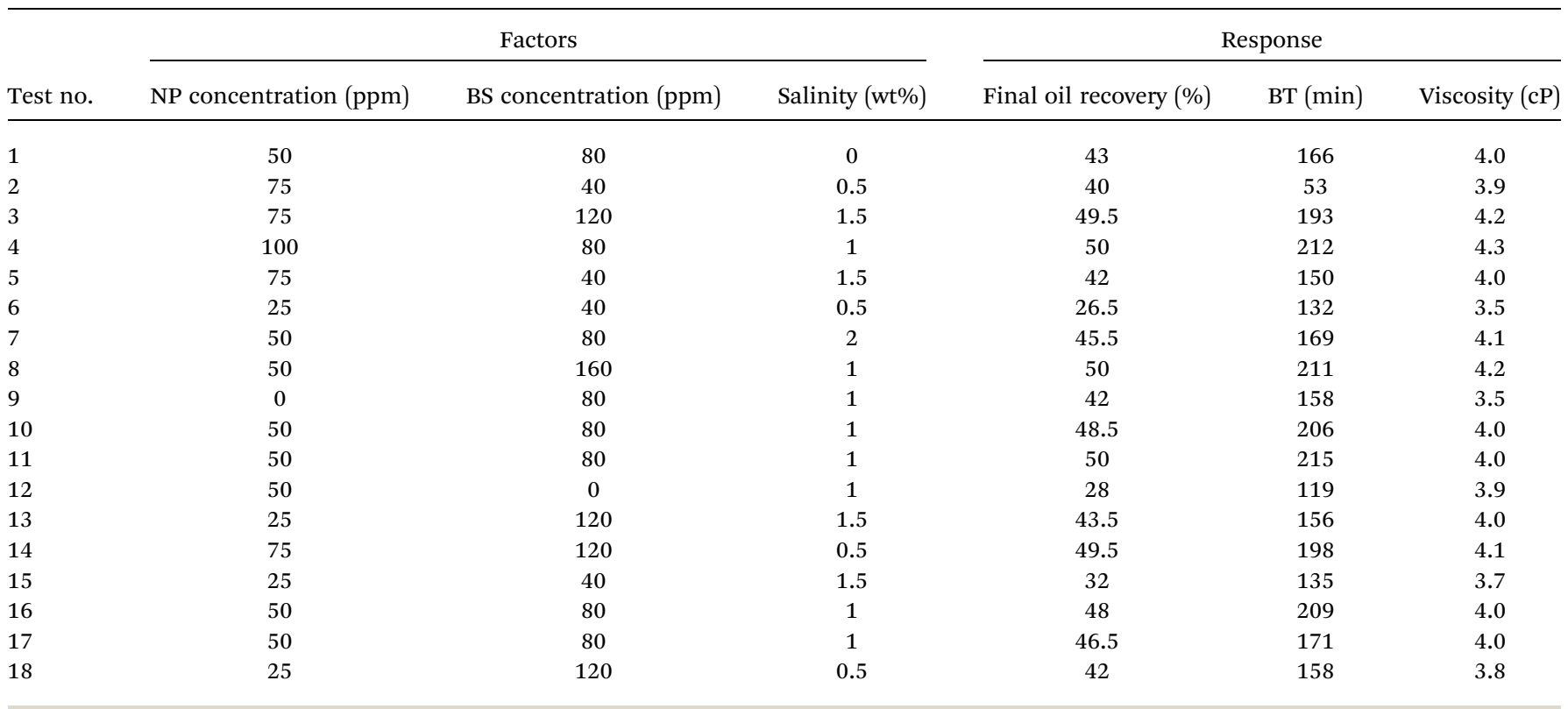

points, and test 4 , respectively. The final oil recovery of the mentioned tests and the IFT values of the related solutions are presented in Fig. 6. The average oil recovery is given for central points. Addition of silica NPs to the solution improved the amount of oil recovery from $42 \%$ in test 9 (without NPs) to an average value of $48.25 \%$ for central points ( $50 \mathrm{ppm} \mathrm{NPs),} \mathrm{which}$ showed $6.25 \%$ enhancement. When a higher concentration of silica was used (100 ppm in test 4), the oil recovery increased from $48.25 \%$ to $50 \%$, thereby showing $1.75 \%$ enhancement. The results indicate that silica NPs are effective in oil recovery. The IFT values of the fluids injected in test 4 , central points, and test 9 were $2.06,2.12$, and $2.39 \mathrm{mN} \mathrm{m}^{-1}$, respectively. As is clearly observed, the solution of test 4 with the least IFT value gives the highest oil recovery. Crude oils with TAN values higher than $0.5 \mathrm{mg} \mathrm{KOH} / \mathrm{g}$ oil are classified as acidic crude oils. ${ }^{31}$ The

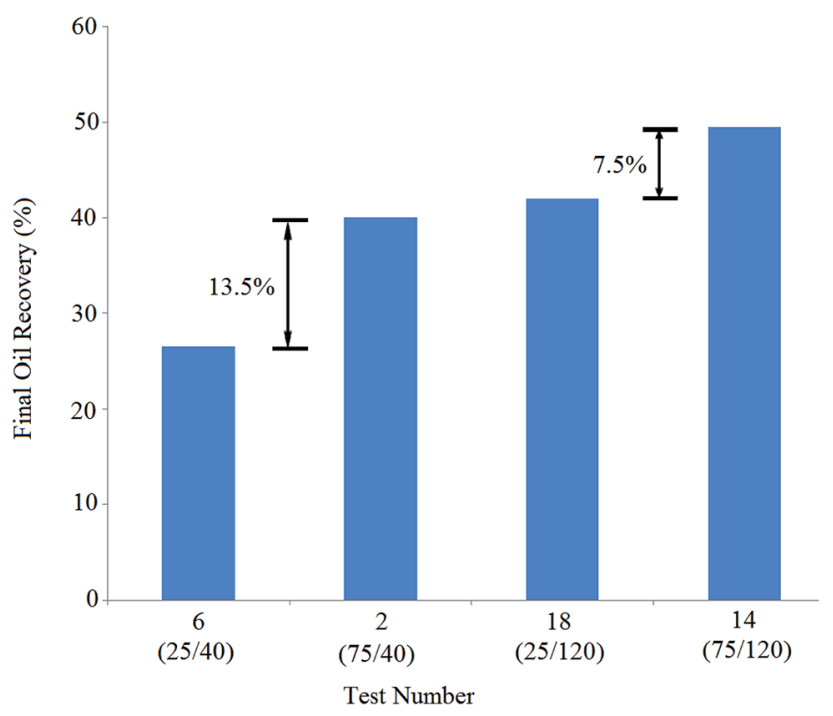

Fig. 5 Final oil recovery for 0.5 wt\% salinity. acidic components present in the crude oil contain many heteroatoms such as oxygen. Therefore, the used crude oil sample with TAN value of $0.46 \mathrm{mg} \mathrm{KOH} / \mathrm{g}$ oil is considered a nearly acidic type with a considerable amount of heteroatoms. Indeed, the reports confirm that the polarity of the head group depends on the molecular structure of the surfactants. ${ }^{30}$ The presence of several hydroxyl groups along with a carboxylate group causes high polarity in the structure of the produced BS, resulting in vigorous interactions with the acidic components of the crude oil. Consequently, the IFT value decreased from 27.16 $\mathrm{mN} \mathrm{m}^{-1}$ (between crude oil and distilled water) to $2.39 \mathrm{mN} \mathrm{m}^{-1}$ for solution 9 (without NPs).

More IFT reduction was observed by increasing the NP concentrations of the central point solutions (50 ppm) and test 4 (100 ppm). NPs can change the IFT value of the surfactant/oil systems due to absorption at the interface. The zeta potentials of BS solution (120 ppm) and spherical silica particles (100 $\mathrm{ppm}$ ) were measured as $-51 \mathrm{mV}$ and $-33 \mathrm{mV}$, respectively, indicating a negative surface charge for both BS molecules and

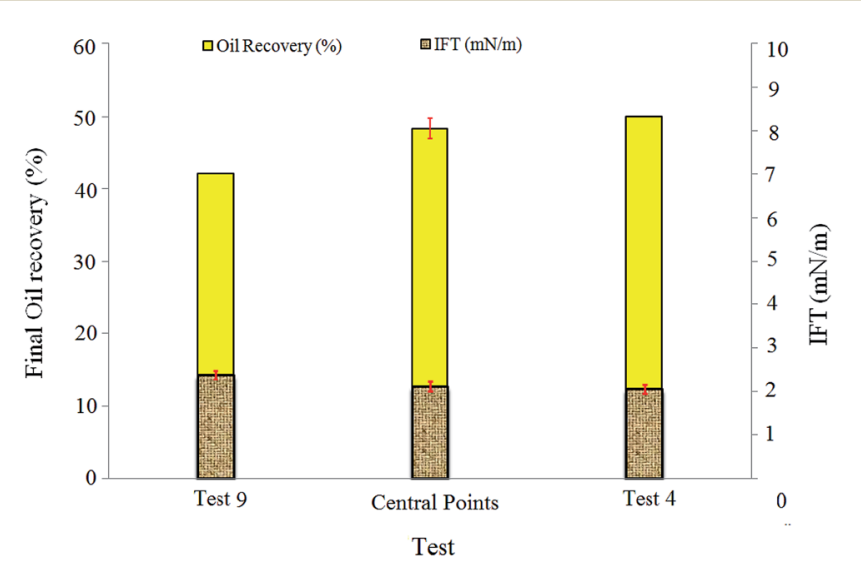

Fig. 6 Final oil recovery and IFT values for 1 wt\% salinity. 
silica NPs. As the NP concentration increased, the repulsive electrostatic forces between NPs and BS molecules promoted the NPs penetration from the bulk of the solution to the wateroil interface. Consequently, the interface became more saturated with NPs and IFT between the injected fluid and residual oil decreased.

The capillary number is defined as a dimensionless number that relates the viscous forces in a system to the capillary forces:

$$
\mathrm{Ca}=\frac{\mu U}{\sigma}
$$

Here, $\mu$ and $U$ are the viscosity and velocity of the displacing fluid, respectively, and $\sigma$ represents IFT between the crude oil and the displacing fluid. ${ }^{11}$

Higher values of this number indicate that viscous forces are more dominant and the capillary forces, which favor oil trapping, are less effective. IFT reduction lowers the capillary forces in the pores and throats and therefore increases the capillary number. ${ }^{11}$ In this case, the injected fluid can penetrate into more pores and throats and release the trapped oil, which will lead to oil recovery improvement. The capillary numbers for tests 9 , central points, and 4 were $7.82 \times 10^{-4}, 1.01 \times 10^{-3}$, and $1.1 \times 10^{-3}$, respectively. The ratio of the capillary number of test 4 to the capillary number of test 9 was almost 1.41 , which indicated $41 \%$ enhancement. After a closer look at the obtained results, it was concluded that the addition of silica NPs has a positive effect on oil recovery by IFT reduction and increasing the capillary number. The BS-NP solution lowered the IFT value between crude oil and aqueous phase, which led to the reduction of the capillary forces in pores and throats. Therefore, the dimensionless capillary number increased and the trapped oil mobilized, which in turn increased the final oil recovery.

The viscosity values of the injected fluids in test 4 , central points, and test 9 (Table 2) were 4.3, 4, and 3.5 times greater than water viscosity $(1 \mathrm{cP})$, respectively. These values show that the displacing fluid viscosity increases as the NP concentration increases. This is due to the formation of three-dimensional and complex networks of silica particles. Moreover, increase in the viscosity of the displacing fluid decreases the viscosity difference between the oil and injected fluid, improving the mobility ratio and delaying $\mathrm{BT}$, which can promote oil recovery from porous media. The final oil recovery and BT of test 9 were $42 \%$ and $158 \mathrm{~min}$, respectively, while the corresponding values for test 4 were 50 and $212 \mathrm{~min}$; this indicates the positive effect of viscosity enhancement on oil recovery.

3.2.3 Flooding tests at $\mathbf{1 . 5} \mathbf{w t} \%$ salinity. The final oil recovery values of tests $3,5,13$, and 15 versus NP/BS concentrations are demonstrated in Fig. 7. All the above-mentioned solutions were flooded at salinity of $1.5 \mathrm{wt} \%$. The solutions of tests 5 and 15 possessed the same BS concentration (40 ppm), but the NP concentration in solution $5(75 \mathrm{ppm})$ was thrice in comparison with that of solution 15 (25 ppm), resulting in 10\% enhancement in oil recovery: oil recovery increased from $32 \%$ (test 15 ) to $42 \%$ (test 5 ).

Indeed, the BS concentrations of the solutions of tests 3 and 13 were equal ( $120 \mathrm{ppm})$, but the NP concentration in solution 3 (75 ppm) was thrice that of solution 13 (25 ppm), resulting in

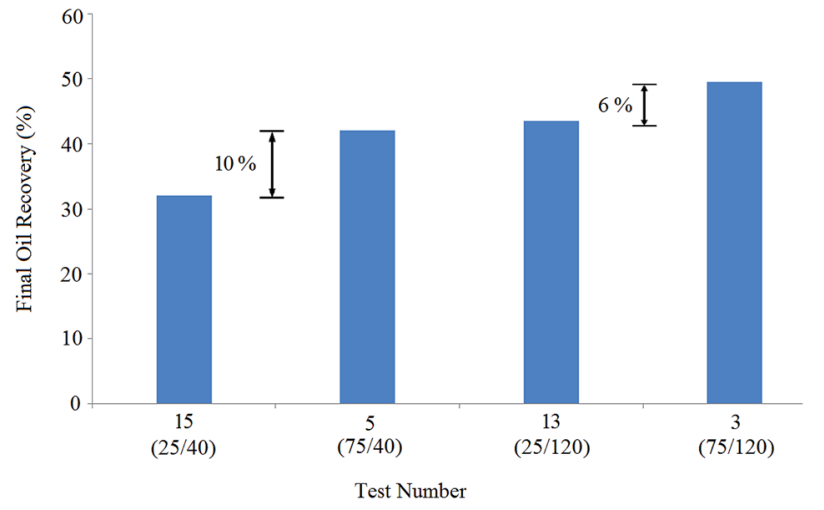

Fig. 7 Final oil recovery for 1.5 wt\% salinity.

$6 \%$ oil recovery enhancement: oil recovery increased from $43.5 \%$ (test 13 ) to $49.5 \%$ (test 3 ).

Considering the obtained results, NPs were efficient in oil recovery enhancement and were more effective at lower BS concentrations. Indeed, the BS concentrations in solutions 3 and 13 were thrice that of solutions 5 and 15 , yielding $7.5 \%$ and $11.5 \%$ more recoveries, respectively: oil recovery increased from $42 \%$ (test 5) to $49.5 \%$ (test 3 ) and enhanced from $32 \%$ (test 15 ) to $43.5 \%$ (test 13 ).

Similarly, the comparison of the pair tests $(3,5)$ and $(13,15)$ shows that increasing the BS concentration positively affects oil recovery, especially at lower NP concentrations. A closer look at the final oil recovery differences $(10 \%, 6 \%, 7.5 \%$, and $11.5 \%)$ shows that the BS concentration enhancement was more effective than NP concentration increase for oil recovery.

3.2.4 The effect of salinity enhancement. The final oil recovery differences for the pair tests of $(6,15),(2,5),(18,13)$, and $(14,3)$ are demonstrated in Fig. 8; in these tests, the salinity difference was $1 \mathrm{wt} \%$ and oil recovery differences were $5.5 \%$, $2.0 \%, 1.5 \%$, and $0.0 \%$, respectively. For pair tests $(6,15)$ and $(2,5)$ with BS concentration of $40 \mathrm{ppm}$, oil recovery differences were more than that of pair tests $(18,13)$ and $(14,3)$ with 120 ppm BS concentration.

This means that BS concentration has an effective role in higher salinities. In other words, the increase in salinity was

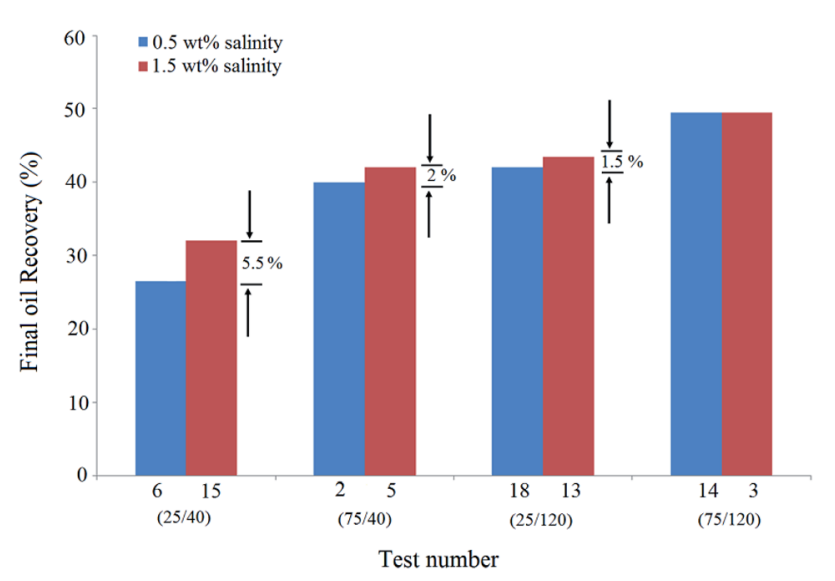

Fig. 8 Final oil recovery difference of pair tests $(6,15),(2,5),(18,13)$ and $(14,3)$. 
more effective at lower BS concentrations. At low BS concentrations, at which the accumulation and adsorption of BS molecules at the interface are not considerable, sodium cations can diffuse and adsorb at the interface easily and lower the electrostatic repulsion between BS and NPs with a negative surface charge. On increasing the BS concentration in the solution, fewer sodium cations can diffuse and adsorb at the interface. As a result, the salinity increase is less effective at higher BS concentrations.

Similarly, the comparison of the pair tests of $(6,15)$ and $(18,13)$ shows that increasing the salinity from $0.5 \mathrm{wt} \%$ to $1.5 \mathrm{wt} \%$ reduces the positive role of BS concentration. Indeed, oil recovery differences of the pair test of $(2,5)$ were $3.5 \%$ less than that of the $(6,15)$ pair test. The NP concentration tripled in these pair tests. It can be concluded that the impact of salinity enhancement is more noticeable at lower NP concentrations. In other words, the increase in salinity reduces the positive effect of NPs on recovery.

3.2.5 Flooding tests at different salinities. The final oil recoveries of test 1 , test 7 , and central points along with the IFT values of the corresponding solutions are presented in Fig. 9. All the above solutions have the same concentrations of BS and NPs, 80 ppm and $50 \mathrm{ppm}$, respectively, but they differ in salinity. As clearly observed in Fig. 9, increasing the salinity from zero (test 1) to $1 \mathrm{wt} \%$ (central points) increased the oil recovery from $43 \%$ to an average value of $48.25 \%$. However, a further increase in salinity from $1 \mathrm{wt} \%$ to $2 \mathrm{wt} \%$ (test 7 ) lowered the oil recovery from $48.25 \%$ to $45.5 \%$, respectively. This means that the salt has a positive effect on oil recovery until an optimum point, after which the addition of the salt has a negative effect.

IFT measurements showed that increasing salinity up to $1 \mathrm{wt} \%$ led to IFT reduction from $3.06 \mathrm{mN} \mathrm{m}^{-1}$ (test 1) to $2.12 \mathrm{mN}$ $\mathrm{m}^{-1}$ (central point tests), but further increase in salinity caused slight increase in IFT $\left(2.27 \mathrm{mN} \mathrm{m}^{-1}\right.$ for test 7$)$.

The capillary numbers for test 1 , central points, and test 7 were $6.98 \times 10^{-4}, 1.01 \times 10^{-3}$, and $9.64 \times 10^{-4}$, respectively. The ratio of the capillary number in central points to the capillary number in test 1 was almost 1.44 . It is obvious that central point experiments with the highest oil recovery compared to tests 1 and 7 resulted in the highest capillary number too.

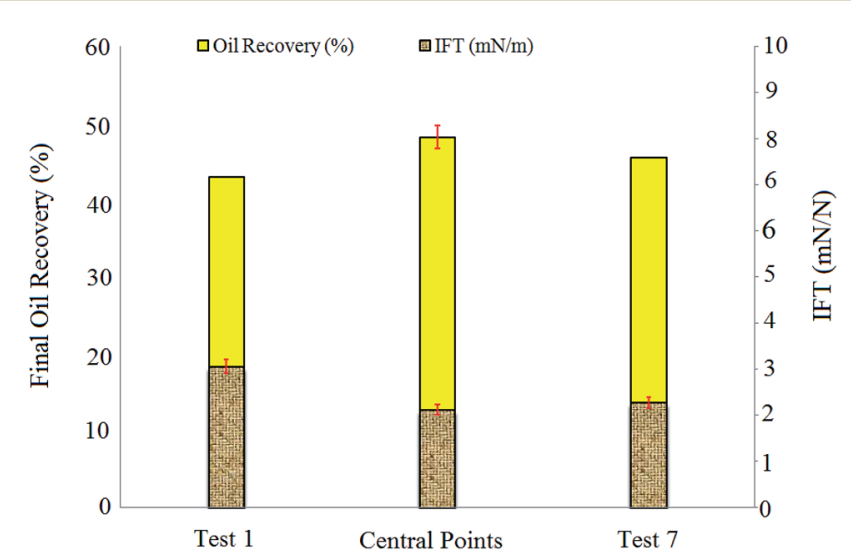

Fig. 9 Final oil recovery and IFT values for test 1, test 7 , and central points.
Initially, the crude oil-aqueous phase interface was negatively charged due to the presence of NPs and anionic BS. After the addition of sodium chloride, some positively charged sodium ions were attracted towards the interface and were placed between BS and NPs. This decreased the electrostatic repulsive forces between negatively charged BS and NPs; also, the electrostatic attraction between the positively charged sodium ions and negatively charged BS and NPs increased. As a result, the effective charge of the interface layer was reduced. Consequently, NPs and BS molecules accumulated easier and were more closely packed at the oil-aqueous phase interface. ${ }^{12,39}$ In other words, the electrostatic effect of the salt changed the distribution of NPs and BS at the interface and led to better interactions between the crude oil-BS hydrophobic chain and the water-hydrophilic head group of BS.

Indeed, the presence of the salt in the aqueous solution increased the movement of BS and NPs from the bulk into the interface. Other sodium ions remained in the bulk of the solution and compressed the electrical double layers around NPs and BS molecules. This increased the electrostatic repulsion between NPs and BS surfaces and promoted their movement to the interface. The salting-out effect of NPs and biosurfactants is another reason for IFT reduction. Salt ions attract some water molecules, which can lower their availability to interact with the BS molecules and NPs in the bulk solution. This accelerates the movement of BS molecules and NPs from the bulk to the interface. ${ }^{12}$

All the above reasons were involved in IFT reduction with increasing salinity. This trend was true until optimum salinity was reached, after which IFT decreased to its minimum value. Beyond this point, a very high sodium ion content prevented the accumulation of NPs and BS molecules at the interface due to hindrance effects. This increased IFT of the solution. Therefore, there was an optimum concentration for salinity to achieve minimum IFT and afford the greatest performance. This is in agreement with the results reported by other researchers. ${ }^{\mathbf{4 0 , 4 1}}$

\subsection{Statistical modeling}

The results of ANOVA are summarized in Table 3. The relatively high $R^{2}(0.9016)$ value indicates that the given model correlates well with the experimental values. The $p$-value (0.0035) implied that the model is significant at a 95\% probability level. The best model was a modified quadratic polynomial to state the relationships between oil recovery, as the response, and the selected parameters.

$$
\text { Oil recovery } \begin{aligned}
(\%) & =-1.37083+0.42058 A+0.49411 B+17.27917 C \\
& -1.31250 A B-0.05500 A C-0.034375 B C \\
& -1.29333 A^{2}-1.59896 B^{2}-4.98333 C^{2}
\end{aligned}
$$

Here, $A, B$, and $C$ denote the NP concentration (ppm), BS concentration (ppm), and salinity (wt\%), respectively. Based on Table 3, BS concentration with the least $p$-value $(0.0002)$ is the most effective factor in oil recovery. It is consistent with the results obtained in Sections 3.1.1 and 3.1.3. BS concentration enhancement at fixed salinity was more effective than increasing NP concentration. Salinity and NP-BS interaction were the next important factors. 
Table 3 ANOVA results

\begin{tabular}{|c|c|c|c|c|c|}
\hline Source & Sum of squares & DOF & Mean square & $F$-value & $p$-value \\
\hline Model & 843.91 & 9 & 93.77 & 8.15 & 0.0035 \\
\hline$A-\mathrm{NP}$ conc. (ppm) & 172.27 & 1 & 172.27 & 14.96 & 0.0048 \\
\hline$C$ - salinity (wt $\%)$ & 13.14 & 1 & 13.14 & 1.14 & 0.3165 \\
\hline$A B$ & 13.78 & 1 & 13.78 & 1.20 & 0.3057 \\
\hline$A C$ & 3.78 & 1 & 3.78 & 0.33 & 0.5823 \\
\hline$B^{2}$ & 142.80 & 1 & 142.80 & 12.40 & 0.0078 \\
\hline$C^{2}$ & 33.86 & 1 & 33.86 & 2.94 & 0.1247 \\
\hline$R^{2}$ & 0.9016 & & & & \\
\hline
\end{tabular}

Fig. 10a shows the interaction effect of NP concentration on oil recovery at BS concentrations of 40 and $120 \mathrm{ppm}$ and constant salinity of $1.0 \mathrm{wt} \%$.

As observed, the oil recovery increased from $26.7 \%$ to $45.1 \%$ with increasing NP concentration from zero (point A) to 100 ppm (point B), respectively, indicating 18.4\% oil recovery enhancement. Indeed, more oil recovery was achieved at a higher BS concentration (120 ppm). In this case, the oil recovery was enhanced from $43.1 \%$ to $51 \%$ with increasing NP concentration from zero (point C) to $100 \mathrm{ppm}$ (point $\mathrm{D}$ ), respectively, indicating $7.9 \%$ increase in oil recovery. This behavior indicates that the effect of NP concentration on oil recovery is greater at lower BS concentrations. It is worth noting that oil recovery enhances more steeply at the BS concentration of $40 \mathrm{ppm}$, which confirms the obtained result. This trend was in agreement with the results obtained in Section 3.1.1, where an increase in NP concentration from $25 \mathrm{ppm}$ to $75 \mathrm{ppm}$ at BS concentrations of $40 \mathrm{ppm}$ and $120 \mathrm{ppm}$ led to $13.5 \%$ and $7.5 \%$ enhancements in oil recovery, respectively.

Fig. 10b shows the interaction effect of the rhamnolipid concentration on oil recovery at two salinity values of 0.5 and $1.5 \mathrm{wt} \%$ and a constant NP concentration of $50 \mathrm{ppm}$. The crossed curves state that the effect of salinity on oil recovery is dependent on the level of BS concentration and vice versa. Indeed, the sharp slopes indicate that the interaction of these two factors is significant. In other words, the addition of the salt has a synergistic effect on the oil recovery from porous media. According to this figure, as the salinity increases, the oil recovery enhances to a maximum value and then decreases. At 0.5 wt $\%$ salinity, with increasing BS concentration, the oil recovery enhances from $22.4 \%$ (point A) to its maximum value (48.1\%) at the BS concentration of $125 \mathrm{ppm}$ and then declines to $47.3 \%$ (point B, BS concentration of $160 \mathrm{ppm}$ ). A similar trend was observed at $1.5 \mathrm{wt} \%$ salinity: the oil recovery increased from $27.1 \%$ (point C) to the highest value of $48.6 \%$ at the BS concentration of $115 \mathrm{ppm}$ and then declined gradually; the plotted graph crosses the other at $125 \mathrm{ppm}, 48.1 \%$. Then, the oil recovery decreased further to $46.4 \%$ (point D, BS concentration of $160 \mathrm{ppm}$ ). It is concluded from this figure that the increasing salinity has a positive effect on oil recovery at BS concentrations less than 125 ppm but beyond this concentration, it adversely affects the process.

The relationship between the BS concentration and salinity is shown by the $2 \mathrm{D}$ contour in Fig. 11. As illustrated in this figure, there is an optimum value for salinity to achieve the maximum oil recovery; this figure is consistent with Fig. $10 \mathrm{~b}$.

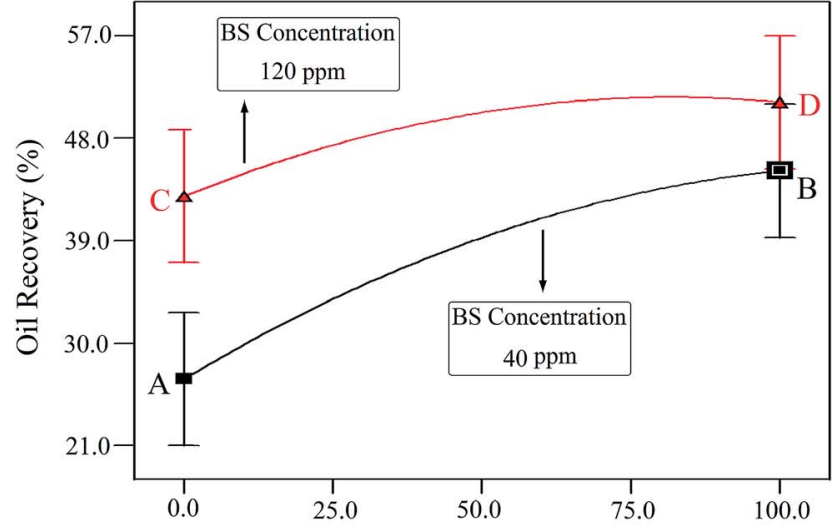

a

Silica NP Concentration (ppm)

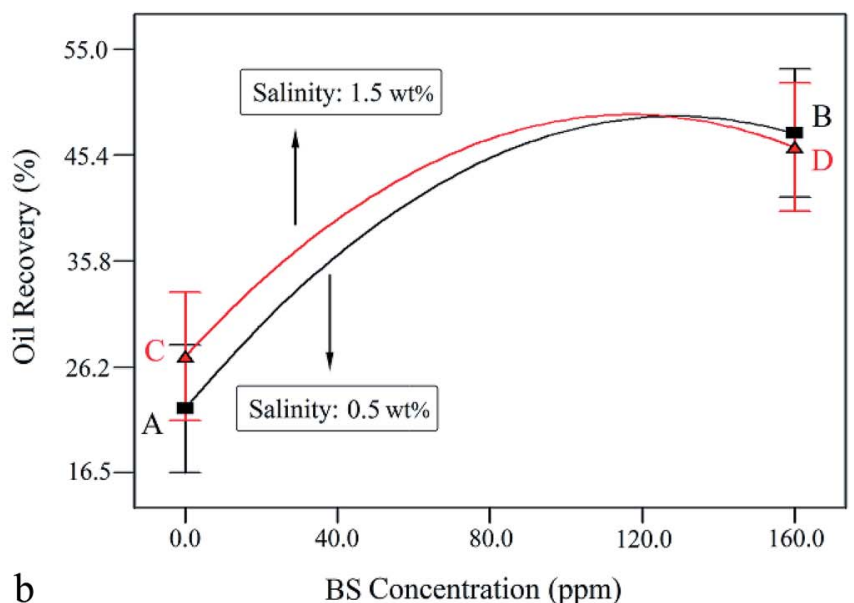

Fig. 10 (a) Interaction of NP and BS concentrations at constant salinity (1 wt\%). (b) Interaction of BS concentration and salinity at a constant NP concentration (50 ppm). 
This behavior was also observed as the BS concentration increased: The oil recovery increased with increasing BS concentration. After the optimum concentration, the oil recovery showed a declining trend when the BS concentration was further increased. Oil recovery reduction after an optimum BS concentration was due to micelle formation, which reduced the concentration of the BS molecules at the interface and decreased their ability to reduce the IFT value. The optimal conditions to reach maximum oil recovery were determined at the NP concentration of $97.5 \mathrm{ppm}$, BS concentration of $110.8 \mathrm{ppm}$, and salinity of $1.17 \mathrm{wt} \%$. Under the optimal conditions, the predicted oil recovery value was $51.2 \%$ and the corresponding oil recovery was $53.4 \%$ (confirmation test). Therefore, the value predicted by the model was very close to the experimental value, indicating good accuracy of the model to optimize the process.

\subsection{The effect of NP morphology (in simultaneous flooding of BS-NPs) on oil recovery}

3.4.1 Micromodel flooding tests. To study the effect of NP morphology (in BS-NP solution) on oil recovery, several micromodel tests were carried out at the optimum point given by the mathematical model using different-shaped NPs (Table 4). Fig. 12 shows the final oil recovery values obtained for these tests. Test 1 represents the confirmation test conducted with spherical NPs, which led to the maximum oil recovery of $53.4 \%$. The synthesized spherical NPs were highly uniform with smooth surfaces (Fig. 1A). This is due to the isotropic growth of particles during synthesis. The highest oil recovery of spherical particles was related to their high uniformity compared to that of other applied NPs, leading to better distribution at the interface and better interactions with crude oil components. Consequently, more IFT reduction and wettability alteration were achieved compared to those for other particles. Indeed, the highest value of the capillary number was obtained for the BSspherical NP solution and it was almost $18 \%$ greater than the capillary number for BS-rod-like NP solution (Table 4). This indicates the greater effect of spherical NPs on oil recovery compared to applied non-spherical particles.

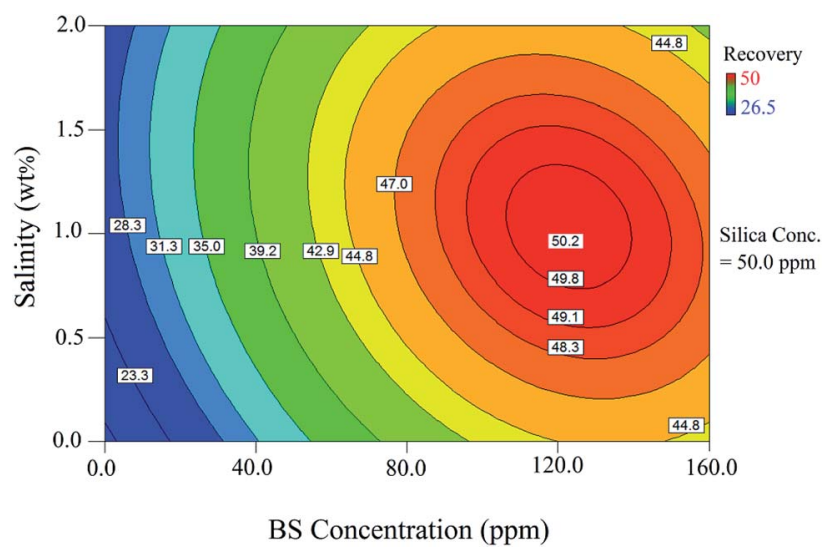

Fig. 11 Contour plot of oil recovery at a constant silica concentration (50 ppm).
Tests 5 and 4 yielded the minimum oil recoveries of $40 \%$ and $44.5 \%$, respectively. One-dimensional nanowires along with irregular-shaped NPs (aspect ratio of 8) were present in the solution of test 4 (Fig. 1B). Rod-like NPs with tetragonal crosssections (aspect ratio of 6) were present in test 5 (Fig. 1E). Low oil recovery in these tests was related to the log-jamming mechanism. NPs, which are smaller than pore throats, accumulated and blocked the rock throats. Moreover, the constant differential pressure and narrowing fluid cross area enhanced the fluid velocity at the pore throats. Water molecules moved faster than silica NPs. Consequently, the accumulation of NPs and pores occurred, and oil recovery decreased. ${ }^{20}$ This is more likely to happen for non-uniform particles with a high aspect ratio compared with spherical particles with high uniformity. The results are shown in Table 4 .

3.4.2 Contact angle measurements. It has been proven that the wettability alteration of porous media is one of the most important factors, among many parameters, which has a great effect on the release of trapped oil through porous media. ${ }^{\mathbf{1 8 , 1 9}}$ Contact angle measurement is a necessary test to investigate the relative degree of solid surface hydrophilicity. ${ }^{32-34,42}$ The contact angles between the glass surface and different BS-NP solutions were measured; the reduction trend $v s$. time is plotted in Fig. 13. It is obvious that the contact angles decrease with time due to the hydrophilicity of the glass surface: the lower the contact angle, the higher degree of the glass surface hydrophilicity. ${ }^{32-34,42}$ Applying NPs along with BS changed the glass surface wettability to a strongly water-wet condition. In each solution, the contact angle decreased to a noticeably lower value within ten minutes. The results are given in Table 4 .

As can be observed, spherical NPs have a greater effect on wettability than non-spherical NPs. The solution of test 1 (Table 4), which reduced the contact angle from $112^{\circ}$ to $8^{\circ}$, exhibited the greatest impact on contact angle $(92.8 \%$ reduction). However, the solution of test 5 , which changed the contact angle from $120^{\circ}$ to $17^{\circ}$ with $85.8 \%$ reduction, showed the least effect on the contact angle.

NPs create a wedge-film structure at the contact region of three phases (oil, fluid, and solid). This film spreads along the solid surface and separates the oil from the solid surface. This mechanism is called the structural disjoining pressure. ${ }^{20}$ When the weight fraction of NPs in the fluid increases, more NPs are located inside the wedge film, leading to a higher disjoining pressure and mobilization of more trapped oil. Additionally, sodium cations present in the injected fluid interact with the anionic acidic components of the crude oil adsorbed on the solid surface, which improves the wettability alteration (ion-binding mechanism). ${ }^{43}$ Indeed, the hydrophobic interactions of the anionic BS molecules with the anionic components of crude oil have a great effect on wettability alteration. All the above factors have synergistic effects on detaching the oil layer from the solid surface and changing the wettability to the water-wet state.

Spherical NPs were more effective in IFT reduction. The higher uniformity of spherical NPs compared to that of other synthesized NPs led to better and more effective dispersion in the base fluid, resulting in more IFT reduction, better wettability alteration, and as a result, more oil recovery. Achieving 
Table 4 Results of oil recovery tests (BS-NP solutions) at optimum point

\begin{tabular}{|c|c|c|c|c|c|}
\hline & Test 1 & Test 2 & Test 3 & Test 4 & Test 5 \\
\hline NP morphology & Spherical & Sponge-like & $\begin{array}{l}\text { Mixture of spherical, } \\
\text { rod-like and irregular NPs }\end{array}$ & $\begin{array}{l}\text { One-dimensional nanowires } \\
\text { and irregular NPs }\end{array}$ & $\begin{array}{l}\text { Rod-like } \\
\text { (tetragonal cross section) }\end{array}$ \\
\hline Breakthrough time (\% PV) & 79 & 76 & 63 & 59 & 57 \\
\hline Breakthrough time (min) & 211 & 201 & 166 & 156 & 151 \\
\hline Final oil recovery (\%) & 53.4 & 47 & 45.6 & 44.5 & 40 \\
\hline IFT $\left(\mathrm{mN} \mathrm{m}^{-1}\right)$ & 1.85 & 1.94 & 2.11 & 2.23 & 2.31 \\
\hline Capillary number $\times 10^{4}$ & 12.7 & 11.8 & 11.1 & 11.0 & 10.8 \\
\hline Initial contact angle $\left(^{\circ}\right)$ & 112 & 113 & 117 & 118 & 120 \\
\hline Final contact angle $\left(^{\circ}\right)$ & 8 & 12 & 15 & 16 & 17 \\
\hline Contact angle change (\%) & 92.8 & 89.3 & 87.2 & 86.4 & 85.8 \\
\hline Viscosity (cp) & 4.4 & 4.3 & 4.4 & 4.6 & 4.7 \\
\hline
\end{tabular}

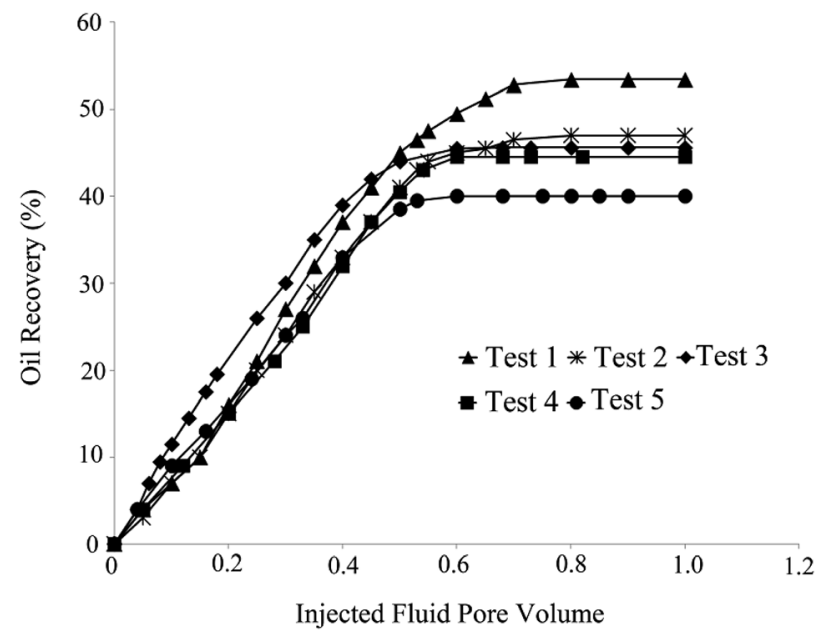

Fig. 12 Oil recovery factor versus injected fluid pore volume for different BS-NP solutions.

wettability alteration to more water-wet state along with other mechanisms including IFT reduction and good mobility ratio is essential for the successful oil recovery process.

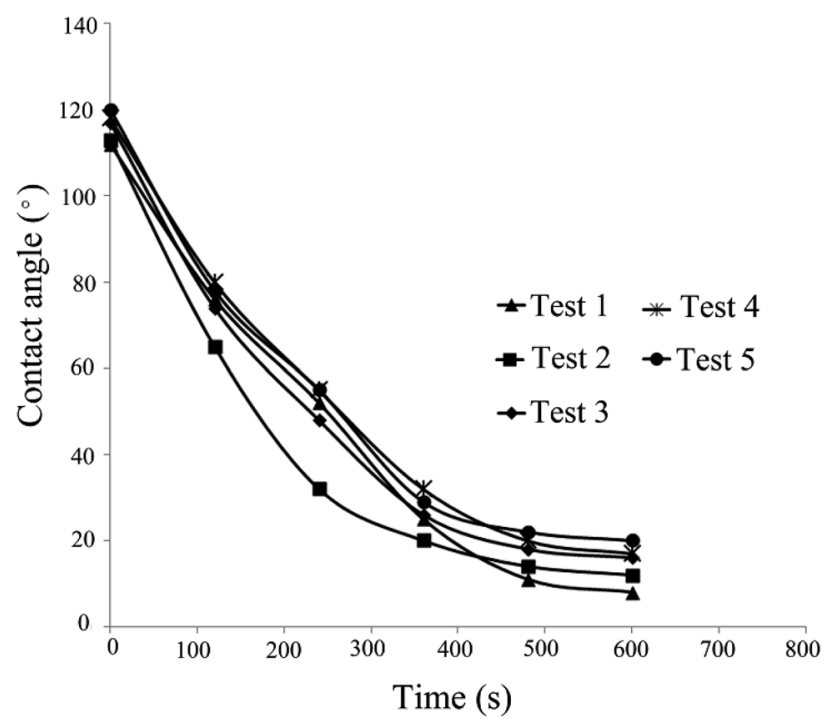

Fig. 13 Contact angle variations for different BS-NP solutions.

\subsection{Core flooding tests}

In order to investigate the effect of the BS-NP solution on oil recovery from the oil-wet carbonate core, two core-flood tests were carried out. BS-NP solutions with spherical and sponge-like particles, which resulted in the highest oil recovery in the micromodel tests (Table 4), were applied in the core flood tests as tests 1 and 2, respectively. Fig. 14 demonstrates the profile of oil recovery versus pore volumes of the injected fluid for four pore volumes of brine injection and four pore volumes of the BS-NP solution injection. It can be observed from this figure that both solutions have a positive impact on oil recovery after brine flooding. As shown in the figure, the increase in oil recovery is almost constant $(\sim 21 \%)$ after two pore volumes of brine flooding, but the total oil recoveries increase to $26.1 \%$ and $25.1 \%$ after the injections of BS-NPs with spherical NPs and sponge-like NPs, respectively. Indeed, the oil recovery is almost constant after three pore volumes of injected BS-NP solution. The results obtained confirmed that BS-NP fluids enhanced the oil recovery from the carbonate rock. In brine flooding, the displacing fluid prefers to pass through large throats and pores with less resistance. Mobility ratio improvement, IFT reduction,

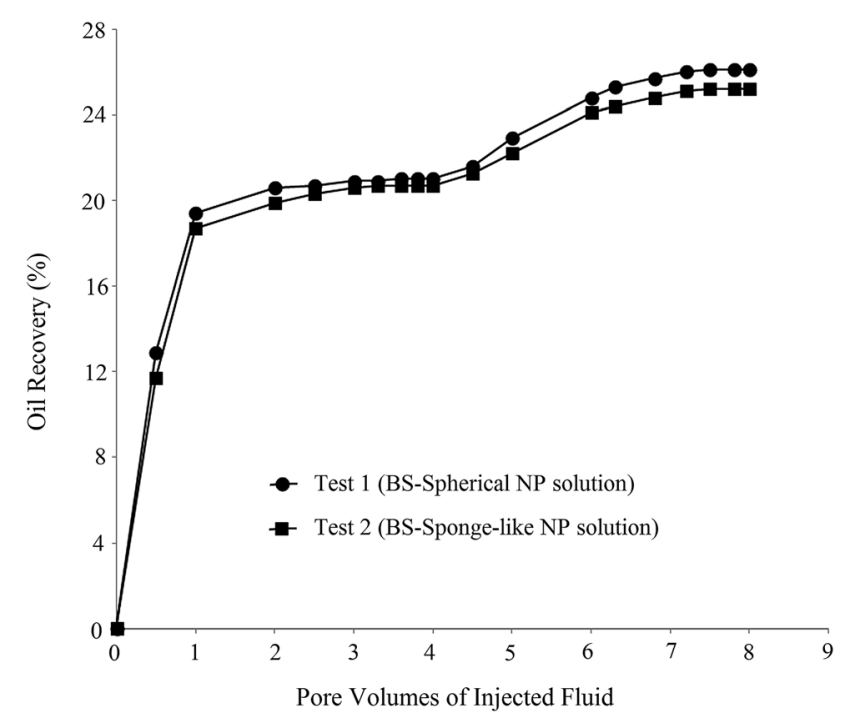

Fig. 14 Oil recovery results for brine flooding and BS-NP flooding of carbonate core sample. 


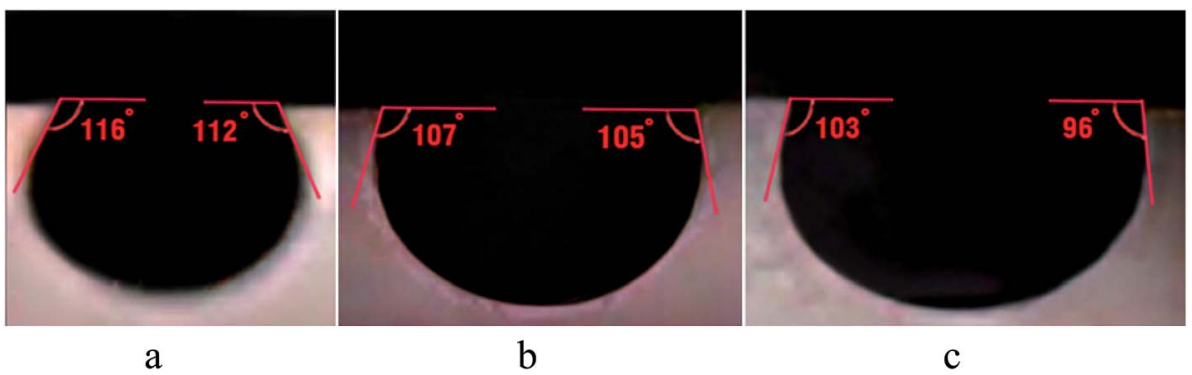

Fig. 15 The measured contact angles of (a) test 1 , (b) test 2, and (c) test 3.

and wettability alteration are the parameters that lead to more oil recovery compared to brine flooding. BS-NP solutions with higher viscosity improved the mobility ratio and decreased the amount of oil bypassed by the displacing fluid.

They also lowered the IFT value and dominated the capillary forces in the pore scale. As a result, the fluid passed through smaller pores with higher resistance and changed the wall wettability to more water-wet states, which increased the oil recovery from porous media. Similar to that observed in the micromodel tests (Table 4), the solution with spherical NPs was more effective for oil recovery compared to the solution with sponge-like particles.

\subsection{Rock wettability alteration}

The contact angles of pellet surfaces were measured to evaluate the effect of BS-NP solutions on rock wettability alteration. BSspherical NP and BS-sponge-like NP solutions, used in core flooding, were applied in these experiments as tests 1 and 2, respectively. The NP concentration in the third solution was considered zero (test 3) to investigate the effect of BS solution (110.8 ppm) without NPs on wettability alteration. The measured initial contact angle between the crude oil droplet and the surface of the carbonate sample was $26^{\circ}$, indicating a strong oil-wet state.

Fig. 15a and b show that the average contact angles of oil on the solid surface are about $114^{\circ}$ and $106^{\circ}$ for tests 1 and 2 , respectively, indicating intermediate water-wet conditions. In these tests, NPs and BS were mainly responsible for wettability alteration from oil-wet to water-wet state. In test 3 , the average contact angle obtained was $99.5^{\circ}$ (Fig. 15c). In this case, the produced BS was mainly responsible for wettability alteration of the rock sample to more water-wet states. The hydrophobic interactions between the tails of anionic BS and adsorbed anionic organic components from oil on the rock surface caused the wettability alteration of the core sample to more water-wet states.

The comparison of Fig. 15a and b with Fig. 15c indicates that silica NPs can alter the wettability of the rock surface to a more water-wet state. The wedge-shaped film structure created by NPs changes the wettability and detaches the oil layer from the surface by the structural disjoining pressure. Indeed, spherical NPs (test 1) are more capable of changing wettability as compared to the sponge-like NPs in test 2 . As previously indicated, high uniformity of spherical particles led to better dispersion in solution and stronger interaction with the adsorbed components on the rock surface, which resulted in more efficient wettability alteration.

A glance at the results obtained from the wettability alteration of glass and carbonate surfaces showed that the BS-NP solutions could change the wettability of both glass and carbonate surfaces from oil-wet to water-wet conditions. It is worth mentioning that a strongly water-wet state was achieved for the glass surface, especially for BS-spherical and BS-spongelike NP solutions; the droplets were almost totally spread out on the surface. However, an intermediate water-wet condition was established for carbonate surfaces. This is due to the nature of solid surfaces: carbonate rock surfaces reflect the real conditions of oil reservoirs much better; the results obtained were more realistic than those obtained from the glass surfaces.

\section{Conclusion}

In this study, simultaneous flooding of a rhamnolipid (anionic BS) and synthesized silica NPs was investigated. Micromodel flooding tests were designed using RSM and carried out using BS-spherical NPs. The effect of variables (NP concentration, BS concentration, and salinity) on oil recovery and their interactions were thoroughly investigated. It was shown that the maximum oil recovery was achieved at an optimum salt concentration. Indeed, NPs exhibited a positive effect on oil recovery, especially at lower BS concentrations. Furthermore, salinity increase was more effective at lower BS concentrations. The results showed that NPs along with biosurfactants resulted in enhancing oil recovery as well as the release of more amounts of trapped oil inside throats and pores due to IFT reduction, wettability alteration of the porous medium, and an increase in the viscosities of the injected fluids as the main mechanisms involved in oil recovery. IFT reduction lowered capillary forces; the BS molecules and NPs could diffuse easily into the pores and throats, altering the wettability. Adding NPs and BS to water resulted in viscosity increase by 3-4 times, which led to a better mobility ratio. To study the effect of NP morphology (in BS-NP solutions) on oil recovery, several tests were conducted with BS and NPs (having different morphologies) at the optimum point predicted by the mathematical model. The results showed that BS-spherical NPs exhibited a better performance than BSnon-spherical NPs in terms of improving oil recovery (53.4\%). The minimum IFT value, $1.85 \mathrm{mN} \mathrm{m}^{-1}$, and the highest change in the glass contact angle, $92.8 \%$, were achieved by the BSspherical NP solution. This means that spherical NPs have 
a greater effect on oil recovery than non-spherical NPs. The high uniformity of spherical NPs, which resulted in better distribution and more effective interactions with crude oil components, was considered as the main reason for this observation. Indeed, the BS-spherical NP solution yielded $26.1 \%$ oil recovery after brine flooding in the core flooding test, which was higher than that of the BS-sponge-like NP solution (25.1\%). Finally, better wettability alteration of the oil-wet carbonate rock was achieved with BS-NP solutions compared to that with the BS solution (without NPs).

\section{Conflicts of interest}

The authors have no conflicts of interest to declare.

\section{References}

1 P. Purswani and Z. T. Karpyn, Laboratory investigation of chemical mechanisms driving oil recovery from oil-wet carbonate rocks, Fuel, 2019, 235, 406-415.

2 F. Moeini, A. Hemmati-Sarapardeh, M. H. Ghazanfari, M. Masihi and S. Ayatollahi, Toward mechanistic understanding of heavy crude oil/brine interfacial tension: The roles of salinity, temperature and pressure, Fluid Phase Equilib., 2014, 375, 191-200.

3 A. Moslemizadeh, A. Shirmardi Dezaki and S. R. Shadizadeh, Mechanistic understanding of chemical flooding in swelling porous media using a bio-based nonionic surfactant, J. Mol. Liq. , 2017, 229, 76-88.

4 S. J. Geetha, I. M. Banat and S. J. Joshi, Biosurfactants: Production and potential applications in microbial enhanced oil recovery (MEOR), Biocatal. Agric. Biotechnol., 2018, 14, 23-32.

5 G. Changhong, Experiences of microbial enhanced oil recovery in Chinese oil fields, J. Pet. Sci. Eng., 2018, 166, 55-62.

6 Y. Al-Wahaibi, S. Joshi, S. Al-Bahry, A. Elshafie, A. Al-Bemania and B. Shibulalb, Biosurfactant production by Bacillus subtilis B30 and its application in enhancing oil recovery, Colloids Surf., B, 2014, 114, 324-333.

7 S. Seo, M. Mastiani, B. Mosavati, D. Peters, P. Mandin and M. Kim, Performance evaluation of environmentally benign nonionic biosurfactant for enhanced oil recovery, Fuel, 2018, 234, 48-55.

8 P. Kanokkarn, T. Shiina, M. Santikunaporn and S. Chavadej, Equilibrium and dynamic surface tension in relation to diffusivity and foaming properties: Effects of surfactant type and structure, Colloids Surf., A, 2017, 524, 135-142.

9 Y. Tu, C. Yang, Y. Cheng, G. Zeng, L. Lu and L. Wang, Effect of saponins on n-hexane removal in biotrickling filters, Bioresour. Technol., 2015, 175, 2231-2238.

10 N. Chegenizadeh, A. Saeedi and X. Quan, Most common surfactants employed in chemical enhanced oil recovery, Petroleum, 2017, 3, 197-211.

11 R. Khademolhosseini, A. Jafari, S. M. Mousavi, H. Hajfarajollah, K. A. Noghabi and M. Manteghian, Physicochemical characterization and optimization of glycolipid biosurfactant production by native strain of Pseudomonas aeruginosa HAK01 and its performance evaluation for MEOR process, RSC Adv., 2019, 9, 7932-7947.

12 N. Saxena, N. Pal, S. Dey and A. Mandal, Characterizations of surfactant synthesized from palm oil and its application in enhanced oil recovery, J. Taiwan Inst. Chem. Eng., 2017, 81, 343-355.

13 T. Song, C. Yang, G. Zeng, G. Yu and C. Xu, Effect of surfactant on styrene removal from waste gas streams in biotrickling filters, J. Chem. Technol. Biotechnol., 2012, 87(6), 785-790.

14 C. Yang, H. Qian, X. Li, Y. Cheng, H. He, G. Zeng and J. Xi, Simultaneous removal of multi-component VOCs in biofilters, Trends Biotechnol., 2018, 36(7), 673-685.

15 Y. Cheng, H. He, C. Yang, G. Zeng, X. Li, H. Chen and G. Yu, Challenges and solutions for biofiltration of hydrophobic volatile organic compounds, Biotechnol. Adv., 2016, 34(6), 1091-1102.

$16 \mathrm{~J} . \quad$ A. Ali, K. Kolo, A. Khaksar Manshad and A. H. Mohammadi, Recent advances in application of nanotechnology in chemical enhanced oil recovery: Effects of nanoparticles on wettability alteration, interfacial tension reduction, and flooding, Egypt. J. Pet., 2018, 27, 1371-1383.

17 N. Moghaddam, A. Bahramian, Z. Fakhroueian, A. Karimi and S. Arya, Comparative study of using nanoparticles for enhanced oil recovery: wettability alteration of carbonate rocks, Energy Fuels, 2015, 29, 2111-2119.

18 S. Al-Anssari, A. Barifcani, S. Wang, M. Lebedev and S. Iglauer, Wettability alteration of oil-wet carbonate by silica nanofluid, J. Colloid Interface Sci., 2016, 461, 435-442.

19 W. Kuang, S. Saraji and M. Piri, A systematic experimental investigation on the synergistic effects of aqueous nanofluids on interfacial properties and their implications for enhanced oil recovery, Fuel, 2018, 220, 849-870.

20 A. I. El-Diasty and A. M. Aly, Understanding the mechanism of nanoparticles applications in enhanced oil recovery, TPS technical Petroleum Services, SPE-175806-MS, 2015.

21 I. Y. Magda, R. M. El-Maghraby, S. M. Saleh and A. Elgibaly, Silica nanofluid flooding for enhanced oil recovery in sandstone rocks, Egypt, Egypt. J. Pet., 2018, 27, 105-110.

22 H. Zhang, T. S. Ramakrishnan, A. D. Nikolov and D. Wasan, Enhanced oil recovery (EOR) driven by nanofilm structural disjoining pressure: flooding experiments and micro visualization, Energy Fuels, 2016, 30, 2771-2779.

23 A. M. S. Ragab and A. E. Hannora, An experimental investigation of silica nano particles for enhanced oil recovery applications, Presented at the SPE North Africa Technical Conference and Exhibition, Cairo, Egypt, SPE-175829eMS, 2015b, 14-16 September.

24 R. Khademolhosseini, A. Jafari and M. H. Shabani, Micro scale investigation of enhanced oil recovery using nano/bio materials, Procedia Mater. Sci., 2015, 11, 171-175.

25 S. Emadi, S. R. Shadizadeh, A. K. Manshad, A. M. Rahimi and A. H. Mohammadi, Effect of nano silica particles on Interfacial Tension (IFT) and mobility control of natural surfactant (Cedr Extraction) solution in enhanced oil 
recovery process by nano-surfactant flooding, J. Mol. Liq., 2017, 248, 163-167.

26 S. Eshraghi, Y. Kazemzadeh, M. Qahramanpour and A. Kazemi, Investigating Effect of $\mathrm{SiO} 2$ Nanoparticle and Sodium-Dodecyl-Sulfate Surfactant on Surface Properties: Wettability Alteration and IFT Reduction, J. Pet. Environ. Biotechnol., 2017, 8, 1-5.

27 M. Mohajeri, M. Hemmati and A. S. Shekarabi, An experimental study on using a nanosurfactant in an EOR process of heavy oil in a fractured micromodel, J. Pet. Sci. Eng., 2015, 126, 162-173.

28 M. Zargartalebi, R. Kharrat and N. Barati, Enhancement of Surfactant Flooding Performance by use of Silica Nanoparticles, Fuel, 2015, 143, 21-27.

29 M. A. Ahmadi and S. R. Shadizadeh, Induced effect of adding nano silica on adsorption of a natural surfactant onto sandstone rock: Experimental and theoretical study, J. Pet. Sci. Eng., 2013, 112, 239-247.

30 L. N. Nwidee, M. Lebedev, A. Barifcani, M. Sarmadivaleh and S. Iglauer, Wettability alteration of oil-wet limestone using surfactant-nanoparticle formulation, J. Colloid Interface Sci., 2017, 504, 334-345.

31 Z. Mingwei, L. Wenjiao, L. Yuyang, D. Caili, W. Xink, Z. Hongda, Z. Chenwei, G. Mingwei, Z. Yue and W. Yining, Study on the synergy between silica nanoparticles and surfactants for enhanced oil recovery during spontaneous imbibition, J. Mol. Liq., 2018, 261, 373-378.

32 D. P. Suhas, T. M. Aminabhavi, H. M. Jeong and A. V. Raghu, Hydrogen peroxide treated graphene as an effective nanosheet filler for separation application, $R S C A d v$., 2015, 5(122), 100984-100995.

33 D. P. Suhas, T. M. Aminabhavi and A. V. Raghu, paraToluene sulfonic acid treated clay loaded sodium alginate membranes for enhanced pervaporative dehydration of isopropanol, Appl. Clay Sci., 2014, 101, 419-429.

34 D. P. Suhas, T. M. Aminabhavi and A. V. Raghu, Mixed Matrix Membranes of H-ZSM5-Loaded Poly(vinyl alcohol) Used in Pervaporation Dehydration of Alcohols: Influence of Silica/Alumina Ratio, Polym. Eng. Sci., 2014, 54(8), 17741782 .
35 S. M. G. Elyaderani and A. Jafari, Microfluidics experimental study in porous media applied for nanosilica/alkaline flooding, J. Pet. Sci. Eng., 2019, 173, 1289-1303.

36 M. I. Bajestani, S. M. Mousavi, A. Jafar and S. A. Shojaosadati, Biosynthesis and physicochemical characterization of a bacterialpolysaccharide/polyamide blend, applied for microfluidics study in porous media, Int. J. Biol. Macromol., 2017, 96, 100-110.

37 A. Akbari, R. Yegani and B. Pourabbas, Synthesis of poly ethylene glycol (PEG) grafted silica nanoparticles with a minimum adhesion of proteins via one-pot one-step method, Colloids Surf., A, 2015, 484, 206-215.

38 A. Akbari, R. Yegani and B. Pourabbas, Synthesis of high dispersible hydrophilic poly ethylene glycol/vinyl silane grafted silica nanoparticles to fabricate protein repellent polyethylene nanocomposite, Eur. Polym. J., 2016, 81, 86-97.

39 M. Saki and A. R. Khaz'ali, Influence of Surfactant Type, Surfactant Concentration, and Salinity on Interfacial Tension of a Brine/Live Oil/Surfactant Fluid System: A Case Study of Iranian Asmari Oil Reservoir, Iranian Journal of Oil \& Gas Science and Technology, 2017, 6(1), 1-12.

40 S. Kumar and A. Mandal, Studies on interfacial behavior and wettability change phenomena by ionic and nonionic surfactants in presence of alkalis and salt for enhanced oil recovery, Appl. Surf. Sci., 2016, 372, 42-51.

41 L. Wang, C. Yang, Y. Cheng, J. Huang, H. He, G. Zeng and L. Lu, Effects of surfactant and $\mathrm{Zn}$ (II) at various concentrations on microbial activity and ethylbenzene removal in biotricking filter, Chemosphere, 2013, 93(11), 2909-2913.

42 Kh. Jarrahian, O. Seiedi, M. Sheykhan, M. Vafaie Sefti and Sh. Ayatollahi, Wettability alteration of carbonate rocks by surfactants: A mechanistic study, Colloids Surf., A, 2012, 410, 1-10.

43 F. Hajibagheri, A. Hashemi, M. Lashkarbolooki and S. Ayatollahi, Investigating the synergic effects of chemical surfactant (SDBS) and biosurfactant produced by bacterium (Enterobacter cloacae) on IFT reduction and wettability alteration during MEOR process, J. Mol. Liq., 2018, 256, 277-285. 